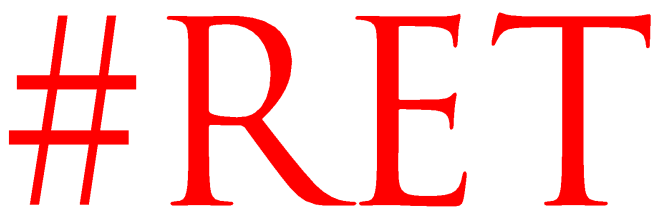

Revista Española de la Transparencia

Núm. 8. Primer Semestre 2019

ISSN 2444-2607. Págs. 121-135

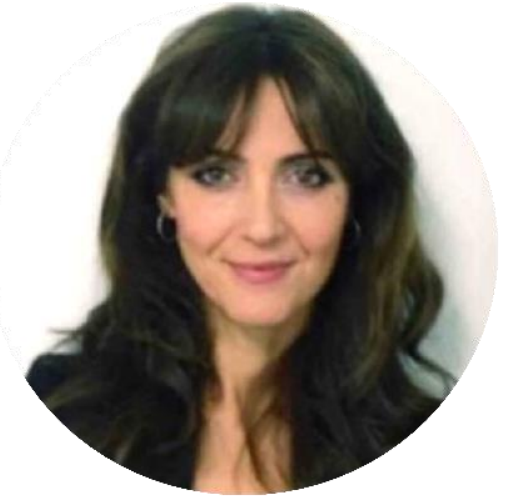

Concepción Campos Acuña Secretaria de Administración Local

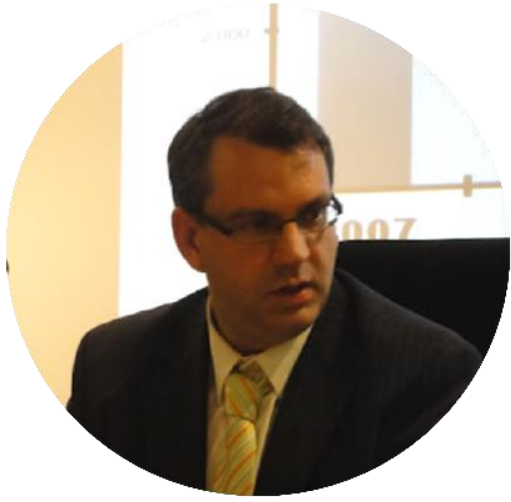

Alberto Vaquero García Universidad de Vigo²

\title{
El portal de transparencia local de Galicia como ejercicio de transparencia desde la administración autonómica y local
}

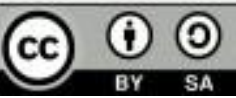

RECIBIDO: 1 de noviembre de 2018 ACEPTADO: 16 de enero de 2019

\footnotetext{
${ }^{1}$ Doctora en Derecho y Licenciada en derecho por la USC, Máster en Derecho de las Administraciones e Instituciones Públicas (USC) y Especialista en Gestión Pública Local (FEMP), es funcionaria de administración local con habilitación de carácter estatal, subescala de Secretaría, categoría Superior, puesto que ha desempeñado en distintos Ayuntamientos, Mancomunidades y Consorcios, en la actualidad es Secretaria de Gobierno Local del Ayuntamiento de Vigo. Codirectora de Red Localis, Coordinadora del GT de Integridad en la Contratación Pública de la Red de Transparencia de la FEMP, Miembro del Consejo Evaluador Externo de la Revista GABILEX y Community Manager de administración electrónica en la Red Social del INAP. Es también autora de numerosos artículos en revistas especializadas, así como directora y autora de diversas monografias sobre gestión pública.

2 Licenciado en Ciencias Económicas y Empresariales, especialidad en Economía Pública, por la Universidad de Vigo, Graduado Social Diplomado por la Universidad Complutense de Madrid, Master en Economía de la Educación y del Trabajo por la Universidad Carlos III de Madrid y doctor en Economia por la Universidad Nacional de Educación a Distancia. Actualmente es profesor e investigador en el Departamento de Economía Aplicada de la Facultad de Ciencias Empresariales y Turismo de la Universidad de Vigo (Grupo GEN de investigación). Ha publicado diversos artículos en revistas indexadas y capítulos de libros en monografías nacionales e internacionales. Es codirector de la Red Localis, Red Local de Administración Pública.
} 


\section{Concepción Campos Acuña y Alberto Vaquero García}

RESUMEN: Uno de los retos más importantes que tienen que afrontar las Administraciones Públicas en los próximos años es adaptarse a la nueva normativa en materia de transparencia y que ésta pase de ser una obligación legal a una cultura con identidad propia en la gestión pública. Sin embargo, los ayuntamientos no siempre cuentan con los medios materiales y humanos suficientes para poder avanzar en el modelo de administración que requiere esa transformación. Si a lo anterior añadimos la reducida dimensión de la planta local en algunas Comunidades Autónomas, como ocurre en Galicia, resulta necesario contar con el apoyo de la Administración Autonómica para poder lograr este objetivo.

En esta comunicación se analizan los esfuerzos realizado por la Xunta de Galicia en materia de transparencia a nivel local en los últimos años, que se ha materializado en un instrumento concreto: el Portal de Transparencia Local de Galicia.

PALABRAS CLAVE: Transparencia, planificación, contratación pública, TIC.

CONTENIDOS: 1. Introducción. - 2. El Portal Autonómico de Transparencia Local de Galicia. - 3. La Guía del Portal Autonómico de Transparencia Local de Galicia. - 4. Conclusiones. - Bibliografía.

\section{The local transparency portal of Galicia as an exercise in transparency from the regional all local administration.}

ABSTRACT: One of the most important challenges facing Public Administrations in the coming years is to adapt to the new regulations on transparency, and also to make transparency move from being a legal obligation to a culture with its own identity in public management. However, city councils do not always have sufficient human and material resources to be able to advance in the model of administration that this transformation requires. If we add to the above the small size of the municipalities in some Regional Government, as in Galicia, it is necessary to have the support of the Regional Administration in order to achieve this objective.

This article analyzes the efforts made by the Xunta de Galicia in terms of transparency at the local level in recent years, which has materialized in a specific instrument: the Local Transparency Portal of Galicia.

KEYWORDS: Transparency, accountability, public information, collaboration, local.. 


\section{El portal de transparencia local de Galicia como ejercicio de transparencia desde la administración autonómica y local}

\section{INTRODUCCIÓN}

En la actualidad, que las Administraciones Públicas proporcionen información a sus ciudadanos es algo natural. La ciudadanía, como financiadora de los bienes y servicios públicos, tiene el derecho a conocer qué hacen los gestores públicos con los fondos que se le asignan y éstos tienen el deber de rendir cuentas de dicha gestión. Para ello, es imprescindible garantizar la máxima transparencia y el acceso a la información a los ciudadanos.

La transparencia tiene que ser uno de los ejes clave en el funcionamiento de cualquier administración pública, siendo esta circunstancia especialmente relevante a medida que se reducen los espacios y fronteras entre sociedad y administración en el escenario de las políticas de gobierno abierto (Cotino, 2013). Únicamente mediante una gestión transparente es posible conseguir una opinión cualificada por parte de la ciudadanía, al mismo tiempo que se potencia su participación, algo cada vez más necesario para mejorar en materia de eficiencia y equidad. Paralelamente es necesario llevar a cabo una adecuada rendición de cuentas, en el marco de las políticas de accountability, siendo más demandadas por la ciudadanía, gracias, en parte, a la aparición de las nuevas tecnologías de la sociedad y de la información que facilitan, de ese modo, el acceso a la información pública y la participación ciudadana e interlocución desintermediada.

Este interés por parte del legislador ha sido evidente en los últimos años, como lo demuestran las numerosas normativas aprobadas, asi como otras iniciativas de autorregulación y la eclosión de órganos de control en esta materia. En el ámbito local gallego, en materia de transparencia, resulta de aplicación la normativa estatal básica, es decir, la Ley 19/2013, de 9 diciembre, de Transparencia, Acceso a la Información Pública y Buen Gobierno (LTBG) y la Ley 1/2016, de 18 de enero, de Transparencia y Buen Gobierno de la Xunta de Galicia (LTBGAL), que, aunque no recoge en su ámbito subjetivo a las entidades locales, sí resulta de aplicación en cierta medida, en cuanto contempla una serie de previsiones que les afectan en relación con la vía autonómica de reclamación previa, según lo dispuesto en la Disposición Adicional Cuarta de la LTBG ${ }^{3}$.

\footnotetext{
3 Disposición adicional cuarta. Reclamación. 1. La resolución de la reclamación prevista en el artículo 24 corresponderá, en los supuestos de resoluciones dictadas por las Administraciones de las Comunidades Autónomas y su sector público, y por las Entidades Locales comprendidas en su ámbito territorial, al órgano independiente que determinen las Comunidades Autónomas. No obstante, lo dispuesto en el párrafo anterior, contra las resoluciones dictadas por las Asambleas Legislativas y las instituciones análogas al Consejo de Estado, Consejo Económico y Social, Tribunal de Cuentas y Defensor del Pueblo en el caso de esas mismas reclamaciones sólo cabrá la interposición de recurso contencioso-administrativo. 2. Las Comunidades Autónomas podrán atribuir la competencia para la resolución de la reclamación prevista en el artículo 24 al Consejo de Transparencia y Buen Gobierno. A tal efecto, deberán celebrar el correspondiente convenio con la Administración General del Estado, en el que se estipulen las condiciones en que la Comunidad sufragará los gastos derivados de esta asunción de competencias.
} 


\section{Concepción Campos Acuña y Alberto Vaquero García}

En este artículo serán objeto de análisis los esfuerzos realizados por la Xunta de Galicia en materia de transparencia a nivel local a partir del proyecto del Portal de Transparencia Local de Galicia (PTLG). Para ello, tras esta introducción, se indican las características más relevantes del citado Portal. En el tercer apartado, se abordan los contenidos básicos de la Guía del PTLG como una herramienta de apoyo a los usuarios del PTLG. Finalmente, se formularán una serie de conclusiones.

\section{EI PORTAL AUTONÓMICO DE TRANSPARENCIA LOCAL DE GALICIA}

Aunque todos los ayuntamientos, en mayor o menor medida, buscan mejorar el nivel de transparencia en su gestión, máxime teniendo en cuenta que las entidades locales constituyen el primer escalón de gobierno y administración, en aplicación del principio de proximidad, en los términos establecidos en la Carta Europea de Autonomía Local, de 15 de octubre de 2015, este objetivo no siempre se consigue.

En no pocas ocasiones, los ayuntamientos gallegos se ven imposibilitados, o cuanto menos limitados, por la falta de recursos materiales y personales, debido, por una parte, al escaso dimensionamiento de su planta.

A lo anterior hay que añadir, además, la reciente implantación de numerosos cambios normativos en ámbitos estratégicos de la gestión pública local, tanto en términos económico-presupuestarios, derivados de la aprobación de la Ley Orgánica 2/2012, de 27 de abril, de Estabilidad Presupuestaria y Sostenibilidad Financiera, y recientemente la vigencia del Real Decreto 424/2017, de 28 de abril, por el que se regula el Régimen Jurídico del control interno en las entidades del Sector Público local; como de procedimiento administrativo y régimen jurídico, en particular, los resultantes de la reforma de 2015, Ley 39/2015, de 1 de octubre, del Procedimiento Administrativo Común de las Administraciones Públicas y Ley 40/2015, de 1 de octubre, de Régimen Jurídico del Sector Público. Asimismo, cabe citar las innovaciones en materia de contratación, con la Ley 9/2017, de 8 de noviembre, de Contratos del Sector Público y en el área de protección de datos, con el Reglamento Europeo de protección de Datos (Piñar, 2014) entre otras modificaciones que, aunque por su visión profundizan en la mejora de la transparencia, suponen una carga excesiva en la gestión diaria respecto a la asignación de medios y prioridades en el cumplimiento de la normativa sobre transparencia.

Sin duda, la especial configuración de la planta local en Galicia, caracterizada por un elevado minifundismo, tiene un claro efecto en esta realidad. En De Diego y Vaquero (2016) se señala como 291 ayuntamientos gallegos (el 93\% del total) poseen una población inferior a 20.000 habitantes. Esta realidad tiene su reflejo en

3. Las Ciudades con Estatuto de Autonomía podrán designar sus propios órganos independientes o bien atribuir la competencia al Consejo de Transparencia y Buen Gobierno, celebrando al efecto un Convenio en los términos previstos en el apartado anterior. 


\section{El portal de transparencia local de Galicia como ejercicio de transparencia desde la administración autonómica y local}

el porcentaje de ayuntamientos (78\%) que se habian decidido a poner en marcha un Portal de Transparencia (Grafico 1).

\section{Gráfico 1: Estado de los portales de transparencia a nivel local (marzo-2017)}

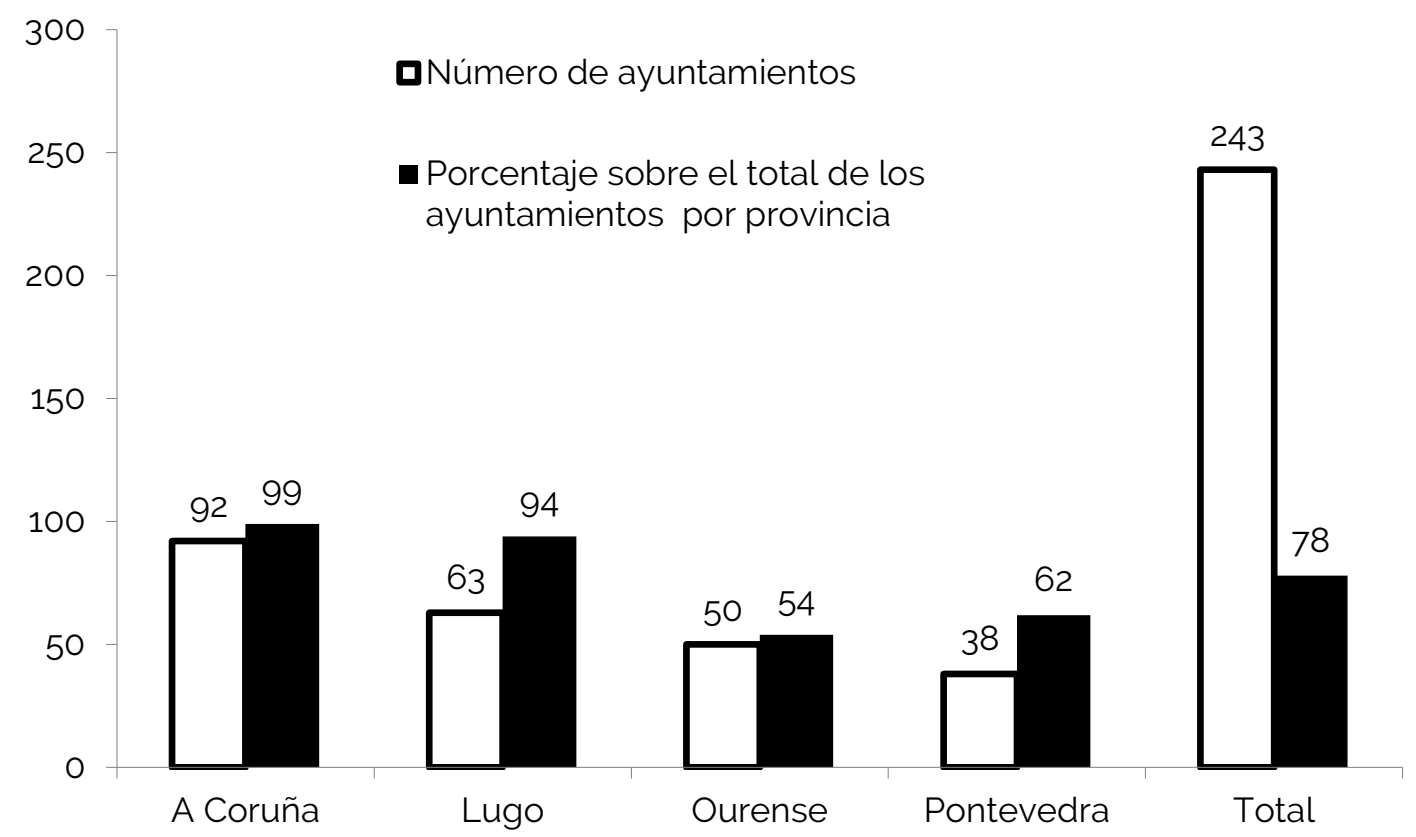

Fuente: Campos y Vaquero (2018)

La Xunta de Galicia ha sido consciente de esta realidad, y en virtud de sus competencias en materia de régimen local y de transparencia, estatutariamente atribuidas, apostó por una iniciativa de carácter colaborativo con los ayuntamientos gallegos: el Portal de Transparencia Local de Galicia (PTLG) ${ }^{4}$. Esta herramienta posibilita una visión general del ejercicio de transparencia a nivel local en Galicia, puesto que la información resulta fácilmente entendible y de sencillo acceso, lo que permite mayores dosis de proximidad a la ciudadania, con la consiguiente ganancia de calidad democrática. Además, el PTLG contempla que aquellas entidades que cuenten con un espacio de transparencia en su web, o incluso con su propio portal de transparencia, lo puedan enlazar directamente. Todas estas

\footnotetext{
${ }^{4}$ Véase el Espacio de Transparencia Local en el portal de Eido Local, portal de Administración Local de Galicia, https://www.eidolocal.gal/ficha-servicio?id=20104. Un resumen de sus contenidos figura en

http://www.fegamp.gal/sites/default/files/como_funciona_o_portal_de_transparencia_de_galicia.p df
} 


\section{Concepción Campos Acuña y Alberto Vaquero García}

acciones las podrán llevar a cabo cumplimentando las diferentes áreas, secciones y subsecciones que se facilitan en la plantilla del PTLG del portal del Eidolocal ${ }^{5}$.

Esta herramienta se pone a disposición de un modo gratuito para los ayuntamientos gallegos, con el objeto que estos den cumplida información a la ciudadanía, posibilitando una vía permanente de comunicación, es decir, los usuarios directos de esta herramienta son los ayuntamientos gallegos. En cuanto la ciudadanía simplemente accederá al Portal de Transparencia del respectivo municipio, en su caso, sin poder entrar al Portal de un modo directo.

El PTLG, surge en diciembre de 2015, como iniciativa de la Vicepresidencia y Consellería de Presidencia, Administraciones Públicas y Justicia de la Xunta de Galicia, en colaboración con la Federación Gallega de Municipios y Provincias (FEGAMP).

En los tres últimos años se observan significativos avances por parte de la Xunta de Galicia en la mejora y desarrollo de esta herramienta. Fruto de este interés, en julio de 2016, se firmó un convenio de colaboración entre la Xunta de Galicia, la FEGAMP y la Universidad de Vigo, para el rediseño del PTLG, con el objetivo de conseguir un mayor aprovechamiento de esta herramienta y mejorar su usabilidad, configurándose como un modelo novedoso en cuanto a las demás experiencias colaborativas que se han ido generando desde la aprobación de la LTBG.

Desde entonces, se ha producido una ingente colaboración con la Xunta de Galicia, en especial con la Dirección General de Administración Local, la Dirección General de la Evaluación y Reforma Administrativa y la Agencia para la Modernización Tecnológica de Galicia (AMTEGA), dada la necesidad de implementación tecnológica de la herramienta y su desarrollo en términos de interoperabilidad y seguridad.

Se puso asi en marcha un proyecto multilateral y diverso en el que se conectaba el know-how del mundo local, a través de la Red Localis, por parte de la Universidad de Vigo, como una red sobre el mundo local de la que forman parte profesores e investigadores universitarios y profesionales del mundo local, con las necesidades de los gestores locales, a través de la FEGAMP.

La Universidad de Vigo, a través de Red Localis, integrada en el Grupo de Investigación GEN (Governance and Economic research Network), ha venido colaborando en el nuevo diseño de portal de transparencia de la Xunta de Galicia para los ayuntamientos gallegos (disponible en la página web eidolocal.gal), para contar con plataforma más completa, eficiente y de fácil utilización.

Esta herramienta permite que los ayuntamientos en Galicia dispongan en un único punto de acceso la información exigida, no sólo por las Leyes de Transparencia estatal y autonómica, sino que también aúna las obligaciones de publicidad activa

\footnotetext{
${ }^{5}$ Dentro de cada sección se podrá acceder a las diferentes subsecciones. En cada una de ellas se encontrará los informes publicados por la entidad o enlaces a otras páginas web donde figure esa información.
} 


\section{El portal de transparencia local de Galicia como ejercicio de transparencia desde la administración autonómica y local}

procedentes de normativa sectorial, como la relativa al empleo público, o el urbanismo y el medio ambiente, como áreas de especial impacto en el ámbito local. Por otra parte, aglutina las recomendaciones de organismos como la Federación Española de Municipios y Provincias (FEMP) y Transparencia Internacional España como buenas prácticas. Además, el PTLG incluye proyección de futuro a través de la incorporación de las políticas públicas de Open Data y Big Data, avanzando así un paso más hacia la reutilización de la información del sector público local, en los términos establecidos en la Ley 37/2007, de 16 de noviembre, de Reutilización de la Información del Sector Público (Curto-Rodríguez, 2017).

Hasta el momento, y pendiente de actualización, han sido más de 40 las entidades locales gallegas que ya han solicitado su adhesión al PTLG, para facilitar asi la gestión de sus obligaciones en materia de transparencia. Si bien el acceso se contempla para todos los ayuntamientos gallegos, el PTLG está pensado sobre todo para aquellos de menor población que, como hemos señalado, representan una parte muy significativa de la planta local municipal en Galicia, al ser éstos los que suelen carecer de personal y recursos materiales, en especial, con dificultades tecnológicas, para llevar a cabo las actuaciones en materia de transparencia.

El PTLG además de suponer una gran ayuda para cumplir con las obligaciones de publicidad activa y ejercicio del derecho de acceso a la información pública (Rams, 2016), es una herramienta que favorece una búsqueda sencilla, estructurada y comprensible de la información (Eido Local, 2018). Asimismo, se ajusta a los parámetros formales establecidos en el artículo 5 de la $\operatorname{LTBGAL}^{6}$, pero con una

\footnotetext{
${ }^{6}$ Artículo 5. Principios generales.

1. Los sujetos enumerados en el artículo 2.1 publicarán de forma periódica y actualizada la información cuyo conocimiento sea relevante para garantizar la transparencia de su actividad relacionada con el funcionamiento y control de la actuación pública.

2. Las obligaciones de transparencia contenidas en este capitulo se entienden sin perjuicio de la aplicación de la normativa autonómica correspondiente o de otras disposiciones específicas que prevean un régimen más amplio en materia de publicidad.

3. Serán de aplicación, en su caso, los límites al derecho de acceso a la información pública previstos en el artículo $14 \mathrm{y}$, especialmente, el derivado de la protección de datos de carácter personal, regulado en el artículo 15. A este respecto, cuando la información contuviera datos especialmente protegidos, la publicidad sólo se llevará a cabo previa disociación de los mismos.
}

4. La información sujeta a las obligaciones de transparencia será publicada en las correspondientes sedes electrónicas o páginas web y de una manera clara, estructurada y entendible para los interesados y, preferiblemente, en formatos reutilizables. Se establecerán los mecanismos adecuados para facilitar la accesibilidad, la interoperabilidad, la calidad y la reutilización de la información publicada, asi como su identificación y localización.

Cuando se trate de entidades sin ánimo de lucro que persigan exclusivamente fines de interés social o cultural y cuyo presupuesto sea inferior a 50.000 euros, el cumplimiento de las obligaciones derivadas de esta Ley podrá realizarse utilizando los medios electrónicos puestos a su disposición por la Administración Pública de la que provenga la mayor parte de las ayudas o subvenciones públicas percibidas.

5. Toda la información será comprensible, de acceso fácil y gratuito y estará a disposición de las personas con discapacidad en una modalidad suministrada por medios o en formatos adecuados de manera que resulten accesibles y comprensibles, conforme al principio de accesibilidad universal y diseño para todos. 


\section{Concepción Campos Acuña y Alberto Vaquero García}

novedad diferencial, y es que concentra en un único portal el conjunto de obligaciones en materia de publicidad activa, con independencia de su fuente obligacional, señaladamente de la normativa básica sobre régimen local, la Ley 7/1985, de 2 de abril, Reguladora de las Bases de Régimen Local, o la normativa en materia de contratos, la Ley 9/2017, de 8 de noviembre, de Contratos del Sector Público (Villoria, 2014).

A través de esta nueva herramienta, los ciudadanos pueden disponer de la información relativa a su ayuntamiento facilitando un ejercicio real de accountability; información que, en materia de publicidad activa, se estructura conforme a la siguiente sistematización de las distintas áreas de actividad de una entidad local: i) institucional, organizativa, de rendición de cuentas, fiscal y patrimonial; ii) de personal; iii) de relevancia jurídica; iv) en materia de contratos; v) de subvenciones, convenios y encomiendas de gestión; vi) de urbanismo y medio ambiente; vii) de planificación, procedimientos y servicios; viii) participación ciudadana y ix) de buen gobierno local.

Complementando al apartado de publicidad activa, el PTLG también dispone de espacio para la publicidad pasiva y el ejercicio del derecho a la información pública, regulado en los artículos 12 y siguientes de la LTBG. (Gutiérrez, 2014) Para ello, esta sección se encuentra divida en los siguientes apartados: i) Presenta tu solicitud7; ii) Consulta el estado de tramitación ${ }^{8}$; iii) Comisión de transparencia9; iv) Preguntas frecuentes ${ }^{10}$ y v) Estadísticas y resoluciones ${ }^{11}$. Además, es necesario señalar que en el PTLG solo estarán visibles los apartados en los que la entidad local publique información.

Finalmente hay que indicar que en el PTLG se incluyen otros apartados como los de: i) aportaciones y sugerencias ${ }^{12}$; ii) Preguntas frecuentes: cómo funciona el portal $^{13}$; iii) Guia rápida del uso del portal ${ }^{14}$; iv) Atención al ciudadano ${ }^{15}$.

\footnotetext{
${ }^{7}$ En este apartado se informa sobre cómo presentar una solicitud de acceso a la información pública a las entidades locales de forma presencial. Para ello se puede descargar el modelo de solicitud, o bien se puede acceder la web o sede electrónica de la entidad local, donde, si está disponible este servicio, se podrá realizar la solicitud en línea.

${ }^{8}$ Se podrá conocer el estado en que se encuentra la solicitud de información, si se cuenta con este servicio en la web de la entidad local.

${ }^{9}$ Figura el enlace a la web de la Comisión de Transparencia de Galicia, entidad que se encarga de solucionar las reclamaciones en materia de acceso a la información pública en la Comunidad Autónoma.

${ }^{10}$ Se disponen de respuestas a preguntas especificas sobre el ejercicio del derecho a la solicitud de información pública.

${ }^{11}$ Son las relativas al cumplimiento del deber de transparencia por parte de la entidad local.

${ }^{12}$ Se facilita información de cómo poder hacer llegar a la entidad local las aportaciones y sugerencias.

${ }^{13}$ Se recoge una amplia batería de respuestas a preguntas generales y especificas relacionadas con el PTLG.

${ }^{14}$ Contiene una guia rápida explicativa de cómo funciona el PTLG.
} 


\section{El portal de transparencia local de Galicia como ejercicio de transparencia desde la administración autonómica y local}

\section{LA GUÍA DEL PORTAL AUTONÓMICO DE TRANSPARENCIA LOCAL DE GALICIA}

Para facilitar el manejo del PTLG, los ayuntamientos que se adhieran dispondrán de un conjunto de utilidades y herramientas entre las que destaca la Guía de Transparencia Local de Galicia $\left(G T L G^{16}\right)$ que se facilitará, exclusivamente, a aquellos ayuntamientos que se adhieran al sistema como un instrumento para su utilización y mejor optimización de las posibilidades que ofrece el Portal ${ }^{17}$.

El objetivo de esta Guía es, además de servir de referencia a los ayuntamientos para el acceso y utilización del PTLG, que toda la ciudadanía conozca las normas sobre transparencia, el ejercicio de sus derechos, la participación de la ciudadanía y la rendición de cuentas por parte de los gestores públicos en el ámbito local (Campos y Vaquero, 2018 y 2019).

La GTLG se adapta a la diferenciación que establece la normativa de transparencia, abordando tanto la publicidad activa, esto es, la información que, de oficio, debe publicarse por mandato legal sin necesidad de solicitud ni identificación, como la pasiva, es decir, el derecho de acceso a la información pública, sometido a un procedimiento de solicitud. Además, la GTLG va más allá de las prescripciones legales, señalando una apuesta clara por el Gobierno abierto, con el objetivo de que la ciudadanía colabore activamente en la mejora de los servicios públicos locales y el fortalecimiento de la labor de transparencia y rendición de cuentas.

Asimismo, La GTLG profundiza en el control, evaluación y seguimiento de las políticas públicas, permitiendo obtener información acerca de si los programas y acciones de las políticas públicas se ajustan a los objetivos previstos o cómo se pueden evaluar sus efectos. La GTLG incluye, además, una serie de recomendaciones prácticas destinadas a la implantación de la transparencia y el avance hacia el gobierno abierto a nivel local.

El Cuadro 1 resume la información contenida en la GTLG. El Cuadro 2 recoge los criterios formales de publicación de esta información.

\footnotetext{
${ }^{15}$ Figura el teléfono de atención al ciudadano en materia de transparencia de la entidad local.

${ }^{16}$ Véase https://www.eidolocal.gal/es/transparencia.

${ }^{17}$ La denominación que figura en la citada web es "Guia práctica de transparencia".
} 


\section{Concepción Campos Acuña y Alberto Vaquero García}

\section{Cuadro 1: Tipo y contenido de la información de la GTLG}

\begin{tabular}{|c|c|}
\hline Tipo de información & Contenido de la información \\
\hline Institucional y organizativa & $\begin{array}{l}\text { La que hace referencia a los miembros del } \\
\text { gobierno municipal, el alcalde o alcaldesa, } \\
\text { concejales y grupos municipales; las funciones } \\
\text { del alcalde, el pleno, la junta de gobierno, los } \\
\text { concejales delegados y los aspectos } \\
\text { organizativos y de funcionamiento, incluyendo } \\
\text { los datos básicos de las entidades } \\
\text { instrumentales del ayuntamiento }\end{array}$ \\
\hline Personal & $\begin{array}{l}\text { Gestión de los recursos humanos, instrumentos } \\
\text { organizativos, relación de puestos de trabajo } \\
\text { (RPTs), cuadro de personal, oferta pública de } \\
\text { empleo, acceso al empleo público y } \\
\text { representación del personal. Esta información } \\
\text { comprende tanto la del ayuntamiento, como de } \\
\text { los organismos descentralizados y sociedades } \\
\text { instrumentales, adscritos al mismo. }\end{array}$ \\
\hline $\begin{array}{l}\text { Presupuestaria, rendición de } \\
\text { cuentas, información fiscal y } \\
\text { patrimonial y de gestión } \\
\text { económica-financiera }\end{array}$ & $\begin{array}{l}\text { Aquella que posibilite un mayor control por } \\
\text { parte de la ciudadania, como presupuestos } \\
\text { iniciales, liquidados, cuentas, información local, } \\
\text { indicadores, información patrimonial }\end{array}$ \\
\hline $\begin{array}{l}\text { Información normativa y de } \\
\text { relevancia jurídica }\end{array}$ & $\begin{array}{l}\text { La que exige la normativa y que puede ser de } \\
\text { interés para la ciudadanía, como ordenanzas y } \\
\text { reglamentos municipales, los bandos de los } \\
\text { alcaldes, la vía de consulta y la participación } \\
\text { ciudadana }\end{array}$ \\
\hline Contractual & $\begin{array}{l}\text { Aquella de obligada publicación, en especial la } \\
\text { relacionada con la actividad contractual } \\
\text { desarrollada por el ayuntamiento en } \\
\text { cumplimento de las normas }\end{array}$ \\
\hline $\begin{array}{l}\text { Subvenciones, convenios y } \\
\text { encomiendas de gestión }\end{array}$ & $\begin{array}{l}\text { Subvenciones concedidas, convenios suscritos } \\
\text { y las encomiendas de gestión }\end{array}$ \\
\hline Urbanismo y al medio ambiente & $\begin{array}{l}\text { La indicada en los términos recogidos en la } \\
\text { normativa sectorial, autonómica y local }\end{array}$ \\
\hline $\begin{array}{l}\text { Planificación, procedimientos y } \\
\text { servicios }\end{array}$ & $\begin{array}{l}\text { Toda aquella que sea necesaria para recoger } \\
\text { esta información }\end{array}$ \\
\hline
\end{tabular}




\section{E portal de transporencia local de Galicio como ejercicio de transparencia desde la administración autonómica y local \\ Cuadro 2: Criterios formales de publicación de la información y contenido de la GTLG}

\begin{tabular}{|l|l|}
\hline \multicolumn{1}{|c|}{ Criterios } & \multicolumn{1}{c|}{ Contenido } \\
\hline Temporales & La información disponible debe estar actualizada \\
\hline Tecnológicos & $\begin{array}{l}\text { La forma de presentar la información debe adecuarse } \\
\text { a la normativa de seguridad e interoperabilidad }\end{array}$ \\
\hline Formato abierto & $\begin{array}{l}\text { La información ha de ser publicada y difundida en } \\
\text { formato electrónico abierto. Además, debe ser } \\
\text { libremente reutilizada }\end{array}$ \\
\hline Accesibilidad & $\begin{array}{l}\text { La información debe ser veraz, de fácil acceso, clara, } \\
\text { sencilla y concisa }\end{array}$ \\
\hline Límites & $\begin{array}{l}\text { La información publicada debe acotarse al derecho } \\
\text { de acceso ante la concurrencia de los límites (artículo } \\
\text { 14 LTBG) y, de manera especial, a lo relativo a la } \\
\text { protección de datos (artículo 15 LTBG) }\end{array}$ \\
\hline
\end{tabular}

Fuente: Campos y Vaquero (2018 y 2019).

A partir de la GTLG, los ayuntamientos conocerán las condiciones para ejercitar el derecho de acceso a la información pública; la resolución de los conflictos por la presencia de límites a ese derecho de acceso a la información; la necesidad de protección de cierto tipo de información que recoge datos de carácter personal; las posibilidades que ofrece la utilización de técnicas disociación y anonimización de la información (Campos y Vaquero, 2018).

También se indican en la GTLG los motivos de inadmisión del trámite de solicitudes, cuál debe ser el procedimiento de acceso, la formalización del citado acceso y la reclamación previa. Asimismo, se recogen otros supuestos de acceso a la información. Finalmente, en la GTLG se recopila información sobre los modelos de evaluación y seguimiento de las obligaciones en materia de transparencia. Este seria, por ejemplo, el contemplado en la ordenanza tipo de transparencia, acceso a la información pública y reutilización de la FEMP; el índice de transparencia de los ayuntamientos (ITA) de Transparencia Internacional España y las recomendaciones prácticas para la aplicación de transparencia (Campos y Vaquero, 2018).

\section{CONCLUSIONES}

La transparencia supone mucho más que cumplir con unas obligaciones normativas, ya que alcanza a un nuevo paradigma de gestión pública, pues contar con una administración transparente garantiza ofertar valor a la ciudadanía y abre innumerables posibilidades para mejorar la calidad de los servicios públicos que prestan las entidades locales (Fernández, Salinas y Salinas, 2013). Por ello, es preciso abordar este cambio desde la necesaria transformación cultural para restaurar la calidad democrática de las instituciones públicas, tan cuestionada por las consecuencias de la crisis económica, y por los casos de corrupción en materia 


\section{Concepción Campos Acuña y Alberto Vaquero García}

de gestión de los últimos años y que han provocado una fuerte desafección política en la ciudadanía (Rubio y Escolano, 2018).

A estos objetivos obedece el PTLG, ya que desde la Xunta de Galicia, como administración autonómica con competencias en materia de régimen local, estatutariamente atribuidas, se es consciente de la necesidad de garantizar mayores dosis de transparencia no sólo a nivel autonómico, sino también en el ámbito local, como entidades más próximas al ciudadano, que prestan un gran número de servicios públicos y constituyen la primera toma de contacto de la sociedad con la administración pública. De ahí que se venga constatando un mayor esfuerzo, especialmente desde la creación del PTLG, una fórmula colaborativa donde la Xunta de Galicia presta asistencia y apoyo a las entidades locales gallegas para que puedan cumplir con sus obligaciones en el campo de la transparencia, tanto derivadas de la normativa estatal como autonómica.

Esta herramienta va más allá de un simple repositorio de información, puesto que su actual diseño permite cumplir no sólo sobradamente las exigencias legales, sino que posibilita la utilización de información para realizar un ejercicio de comparación municipal, precisamente una de las asignaturas pendientes en el ámbito local gallego.

Debe destacarse que el nuevo diseño del PTLG se presenta como un modelo fruto de la colaboración entre la Xunta de Galicia, FEGAMP y la Universidad de Vigo, aspecto este que, constituye una singularidad en el ámbito de los modelos colaborativos sobre transparencia, en particular, por la colaboración tripartita. Para este ejercicio, se cuenta con la Universidad de Vigo como red experta que soporta el diseño del modelo, frente a otras alternativas, donde esto no se ha producido. Este seria el caso del Portal de Transparencia en la nube para entidades locales, creado por el Ministerio de Políticas Territorial y Función Pública ${ }^{18}$, el Portal de Transparencia del Consorcio de Administración Abierta de Cataluña ${ }^{19}$ o el de la Diputación de Palencia ${ }^{20}$. Lo anterior permite, además, un claro ejercicio de transferencia del conocimiento desde la Universidad a las instituciones públicas locales y autonómicas.

Asimismo, se cuenta con la GTLG, de indudable utilidad tanto para los gestores públicos como para la ciudadanía, que se presenta como una potente herramienta

\footnotetext{
18 Su objetivo es la creación del Portal de la Transparencia a las Entidades Locales para aquellas entidades que se suscriban, además de generar toda la infraestructura TIC para su correcto alojamiento en base a lo dispuesto en la LTBG, https://administracionelectronica.gob.es/ctt/transparencia

19 Este portal, donde participan la Generalitat de Cataluña, las cuatro diputaciones provinciales catalanas, la Asociación Catalana de Municipios y Provincias y la Federación de Municipios de Cataluña, tiene como objetivo que las entidades locales catalanas dispongan de un Portal de Transparencia. https://www.aoc.cat/serveis-aoc/transparencia/

${ }^{20}$ https://www.diputaciondepalencia.es/sitio/transparencia-participacion
} 


\section{El portal de transparencia local de Galicia como ejercicio de transparencia desde la administración autonómica y local}

para manejar correctamente la información suministrada por los ayuntamientos gallegos.

En consecuencia, gracias al PTLG y la GTLG, Galicia mejora notablemente la difusión de la información a nivel municipal. Además, con ambos instrumentos se garantiza el derecho de información de la ciudadanía en relación a la organización y funcionamiento de los ayuntamientos en Galicia. Adicionalmente, y esta es una de las grandes novedades, el PTLG se configura como un canal abierto a la participación ciudadana, avanzando asi en las políticas de gobierno abierto.

Sin embargo, todavía hay un importante trabajo por realizar en materia de transparencia municipal en Galicia. Además del rediseño del PTLG (y su posterior desarrollo con la GTLG), que ha contado en su elaboración con las opiniones y valoraciones de los ayuntamientos, a través de un proceso participativo abierto al conjunto de los municipios gallegos, en aras de conseguir un portal de transparencia más fácil de utilizar y que posibilite un mayor y mejor potencial de recopilación de información desde la Xunta de Galicia, es necesario abordar una amplia batería de medidas operativas que refuercen esta visión estratégica.

Entre ellas, destacan la de mejorar la información a los ayuntamientos sobre la potencialidades y ventajas de seguir divulgando la transparencia, en especial, a través de Eido Local, así como la realización de jornadas de formación sobre transparencia en la Escuela Gallega de Administración Pública (EGAP), acercando este proyecto tanto a los empleados públicos municipales, como a los gestores políticos, para facilitar su conocimiento de la herramienta y su posterior adhesión.

Por lo tanto, es evidente que durante los próximos años la transparencia va a ser una de las actuaciones prioritarias a nivel local desde la Xunta de Galicia, desde una visión holística y global, no sólo pensando en la obligatoriedad de publicar y tener accesible la información a la ciudadanía. Además, esta actividad permitirá una mayor participación de la ciudadanía en el gobierno, orientado, en última instancia a una total implantación de las políticas de gobierno abierto sobre los tres ejes que lo definen: transparencia, participación y colaboración. 


\section{Concepción Campos Acuña y Alberto Vaquero García}

\section{BIBLIOGRAFÍA}

Campos Acuña, C. y Vaquero Garcia, A. 2015. "Claves para el gobierno local (20152019). Diez retos de futuro para la administración local", Documento de Trabajo 1/2015 de la Red Localis.

Campos Acuña y Vaquero García, A. 2018. "Avances en materia de transparencia municipal en Galicia: el Portal de Transparencia Local", El consultor de los ayuntamientos, octubre.

Campos Acuña, C. y Vaquero García, A. 2019. "El estado de los portales de transparencia de los ayuntamientos gallegos", Dereito: revista xurídica da Universidade de Santiago de Compostela, vol. 27 (2), en prensa.

Cotino Hueso, L. 2013. Derecho y gobierno abierto. La regulación de la transparencia y la participación y su ejercicio a través del uso de las nuevas tecnologías y las redes sociales por las administraciones públicas. Propuestas concretas, Monografias de la Revista Aragonesa de Administración Pública, pp. 592.

Curto-Rodríguez, R. 2017. "Los portales autonómicos de datos abiertos y la información relacionada con la rendición de cuentas. Punto de partida y situación tras la entrada en vigor de la Ley de acceso a la información pública y buen gobierno", Revista Española de Transparencia, 5, segundo semestre, 80-93.

De Diego Gómez, A. y Vaquero García, A. 2016. "Redimensionamiento de la planta local. Retos frente al minifundismo de Galicia, Documento de Trabajo 5/2016 de la Red Localis.

Fernández Llera, R., Salinas Jiménez, J. y Salinas Jiménez, Ma. 2013. "Transparencia y rendición de cuentas de las entidades locales" XXV Reunión de Estudios Regionales, Oviedo.

Gutiérrez David. E. 2014. "Derecho de acceso a la información pública. Eunomía", Revista en Cultura de la Legalidad, 6, 186-196.

Piñar Mañas, J.L. 2014. "Transparencia y derecho de acceso a la información pública. Algunas reflexiones en torno al derecho de acceso en la Ley 19/2013, de transparencia, acceso a la información y buen gobierno" Revista catalana de dret públic, 49, 1-19.

Rams Ramos, L. 2016. "El procedimiento de ejercicio del derecho de acceso a la información pública", Revista General de Derecho Administrativo, 41.

Rubio Núñez, R. y Valle Escolano, R. 2018. "Implantando la transparencia en el ámbito local: hablan los protagonistas", Ilemata-Revista Internacional de éticas aplicadas, 27, 311-331. 


\section{El portal de transparencia local de Galicia como ejercicio de transparencia desde la administración autonómica y local}

Villoria, M. 2014. "La publicidad activa en la Ley de Transparencia, acceso a la información y buen gobierno: posibilidades e insuficiencia" Govern Obert. Generalitat de Catalunya. 